OPEN ACCESS

Edited by:

Etienne Giraud,

Institut National de la Recherche

Agronomique, France

Reviewed by:

Stephen Peter Kidd,

University of Adelaide, Australia

Barbara C. Kahl,

University Hospital Münster, Germany

*Correspondence:

Lone Gram

gram@bio.dtu.dk

Specialty section:

This article was submitted to Antimicrobials, Resistance

and Chemotherapy,

a section of the journal

Frontiers in Microbiology

Received: 01 March 2016 Accepted: 23 June 2016

Published: 05 July 2016

Citation:

Curtis TD, Gram L and Knudsen GM (2016) The Small Colony Variant of Listeria monocytogenes Is More

Tolerant to Antibiotics and Has Altered Survival in RAW 264.7 Murine

Macrophages.

Front. Microbiol. 7:1056.

doi: 10.3389/fmicb.2016.01056

\section{The Small Colony Variant of Listeria monocytogenes Is More Tolerant to Antibiotics and Has Altered Survival in RAW 264.7 Murine Macrophages}

\author{
Thomas D. Curtis, Lone Gram* and Gitte M. Knudsen \\ Gram Lab, Department of Systems Biology, Technical University of Denmark, Kongens Lyngby, Denmark
}

Small Colony Variant (SCV) cells of bacteria are a slow-growing phenotype that result from specific defects in the electron transport chain. They form pinpoint colonies on agar plates and have a variety of phenotypic characteristics, such as altered carbon metabolism, decreased toxin and lytic enzyme production, aminoglycoside resistance, and increased intracellular persistence. They are clinically relevant in Staphylococcus aureus and Pseudomonas aeruginosa, serving as a reservoir for recurrent or prolonged infections. Here, we found that a SCV mutant in the foodborne pathogen Listeria monocytogenes (strain SCV E18), similar to the high persister mutant phenotype, survived significantly better than the wild type when exposed over a 48-h period to concentrations above Minimal Inhibitory Concentration for most tested antibiotics. SCV E18 survived more poorly than the wildtype in unactivated RAW264.7 macrophage cells, presumably because of its reduced listeriolysin O expression, however, it survived better in reactive oxygen species producing, phorbol 12-myristate 13-acetate-activated macrophages. Although SCV E18 was sensitive to oxygen as it entered the stationary phase, it was significantly more tolerant to $\mathrm{H}_{2} \mathrm{O}_{2}$ than the wild type, which may result from a shift in metabolism, however, further investigation is needed to resolve this. SCV E18 is a spontaneous mutant with a point mutation in the hemA gene. A wild type copy of hemA was complemented on plasmid pSOG30222, which restored the wild type phenotype. The results reported here suggest that the SCV of $L$. monocytogenes could be of clinical importance and highlight a need for adequate clinical screening for this phenotype, as it could affect antibiotic treatment outcomes.

Keywords: Listeria monocytogenes, small colony variants, antibiotic tolerance, persister cells, cell invasion, oxidative stress

\section{INTRODUCTION}

Listeria monocytogenes is a Gram-positive, foodborne pathogen that can cause the rare, but often lethal infection listeriosis. This typically affects pregnant women, neonates, the elderly and the immunocompromised, and can cause mortality rates of up to $34 \%$ (Farber and Peterkin, 1991). Upon entry into the gastrointestinal tract, L. monocytogenes infects host epithelial cells 
and immediately escapes the phagosome where it is free to replicate within the cytosol. Using actin-mediated mobility it spreads to neighboring cells and eventually enters the bloodstream, causing systemic listeriosis if unchecked by the innate immune system (Portnoy et al., 2002). Macrophages are a key part of the innate immune response and control the initial infection by engulfing and killing the bacteria with of a variety of antimicrobial compounds, such as reactive oxygen and nitrogen species (Shaughnessy and Swanson, 2007).

Treatment options for listeriosis are limited as many antibiotics are only bacteriostatic against L. monocytogenes. Ampicillin is the treatment of choice, and is sometimes used in combination with gentamicin, although the necessity of this supplemental treatment has been debated (Temple and Nahata, 2000; Mitjà et al., 2009). For patients who cannot tolerate ampicillin, a combination of trimethoprim and sulfamethoxazole, known as co-trimoxazole (SXT), or macrolides are substituted. (Temple and Nahata, 2000). While resistance in L. monocytogenes is uncommon, particularly for the clinically relevant antibiotics (Morvan et al., 2010), we recently demonstrated that this bacterium can form multi-antibiotic tolerant persister cells (Knudsen et al., 2013). However, it is not known if such cells are formed in vivo during infection, which could further complicate antibiotic treatment.

Persister cells are a very small subpopulation of bacteria that enter a dormant-like state making them refractory to most antibiotics. Their tolerance to antibiotics is not genetically inherited, and can be operationally defined by a biphasic killing curve when treated with bactericidal antibiotics and no observed increase in the Minimum Inhibitory Concentration (MIC) (Lewis, 2010). Persister cells are thought to exist in all bacteria and have been linked to recurrent infections with a number of diseases (Maisonneuve and Gerdes, 2014). Their formation can be either stochastic and the result of phenotypic switching from normally growing cells to those with reduced growth rates (Balaban et al., 2004), or they can be induced by certain conditions, such as biofilms (Lewis, 2010) or internalization by macrophages (Helaine et al., 2014). One of the primary genetic mechanisms of persister formation identified so far are toxin/antitoxin (TA) modules, which consist of a stable toxin that slows bacterial growth or metabolism and an unstable antitoxin that neutralizes the activity of the toxin under growth conditions. By repeatedly exposing Escherichia coli to high concentrations of ampicillin, Fridman et al. (2014) found that bacteria can also tolerate antibiotics by extending the lag-phase by mutations in a variety of pathways, essentially rendering the cells dormant until the transient antibiotic pressure is removed.

Another potential reservoir of persistent and recurrent bacterial infections is the so-called Small Colony Variant (SCV) (Kahl et al., 2016). The SCV is a slow growing phenotypic variant that forms pinpoint colonies when plated on agar, and is the result of either thymidine auxotrophy caused by mutations in the thymidylate synthase gene (Besier et al., 2007; Chatterjee et al., 2008) or an interruption in the electron transport chain (ETC), specifically resulting from an absence of menadione or hemin biosynthesis and metabolism. These variants can either be fixed, which is the result of a mutation in one of the biosynthesis or metabolism genes for the aforementioned compounds, or transient, alternating between the wild type and SCV phenotypes during replication (Edwards, 2012). Additional characteristics include altered carbon metabolism, reduced toxin and lytic enzyme production, and resistance to aminoglycosides (Proctor et al., 2014). Aminoglycosides have also been shown to select for the SCV phenotype, as the reduction in the membrane potential resulting from the disrupted ETC will lower the active transport necessary for aminoglycosides to cross the membrane (Balwit et al., 1994). The combination of trimethoprim/sulfamethoxazole (co-trimoxazole) has been found to select specifically for thymidine auxotrophic SCVs due to the anti-folate action of the drug (Garcia et al., 2013). While SCVs have been primarily studied in Staphylococcus aureus, they have been described in a number of bacteria including Pseudomonas aeruginosa, Eacherichia coli, Vibrio cholera, Salmonella, and Lactobacillus acidophilus (Proctor et al., 2006). Furthermore, they have been linked to a number of recurrent infections including those caused by S. aureus (Proctor et al., 1995), P. aeruginosa (Haussler et al., 1999) and E. coli (Roggenkamp et al., 1998; for a comprehensive review see: Kahl et al., 2016). Although typically less virulent due to their slower growth and reduced expression of virulence factors, such as toxins and lytic enzymes, their ability to adhere, invade and persist within the host cell increases, which is thought to be a crucial factor in the ability of SCVs to cause recurrent or persistent infections (Sendi and Proctor, 2009).

We have previously found that exposure to sub-lethal concentrations of triclosan with subsequent selection on gentamicin could generate stable L. monocytogenes SCV cells (Christensen et al., 2011), which all had mutations in one of the heme biosynthesis or metabolism genes and exhibited the same traits observed in SCVs of other species (e.g., colony size, decreased haemolytic activity, aminoglycoside resistance and altered carbon utilization). Our SCV E18 strain also showed reduced growth typical of SCVs from other species, taking roughly four more hours to reach the same maximal cell density as the wild type strain $\left(\sim 10^{9} \mathrm{CFU} / \mathrm{ml}\right)$, however, we also observed a sensitivity to aerated growth conditions (Kastbjerg et al., 2014), which to our knowledge has not been shown in SCVs from other species.

The phenotypic switching of the SCV and the persister cell are thought to be part of a bacterial bet-hedging strategy, allowing a small percentage of the population to survive and repopulate following stress exposure (Sousa et al., 2012). These two phenotypes share a number of other characteristics including slowed growth, intracellular persistence and a link to chronic infections. Thus, given these similarities, we speculate that a stable L. monocytogenes heme deficient SCV would show a similar tolerance toward a broad range of antibiotics that persister cells do. Furthermore, we sought to determine if L. monocytogenes SCVs are better at invading and surviving intracellularly, as they have been shown to in S. aureus (Sendi and Proctor, 2009). Taken together, these results will help to determine the clinical significance of Small Colony Variants in L. monocytogenes. 


\section{MATERIALS AND METHODS}

\section{Bacterial Strains and Growth Conditions}

Listeria monocytogenes strain N53-1 and a mutant thereof (E18) were included in the present study. N53-1 represents a foodprocessing plant persistent molecular subtype and was isolated from a fish smokehouse (Wulff et al., 2006). Strain E18 [denoted as strain (1) 1-1 in Christensen et al. (2011)] is a stable SCV of N53-1, with a Single Nucleotide Polymorphism (SNP) in the glutamyl tRNA reductase (hemA) gene (Kastbjerg et al., 2014), generated through an adaptive evolution experiment where N531 was exposed to sub-lethal levels of triclosan and subsequently selected on gentamicin (Christensen et al., 2011). Strains were stored at $-80^{\circ} \mathrm{C}$ and grown on Brain Heart Infusion (BHI; Oxoid $\mathrm{CM} 1135$ ) agar at $37^{\circ} \mathrm{C}$ for $24 \mathrm{~h}$. Overnight cultures were achieved by inoculating single colonies in $10 \mathrm{ml}$ of BHI broth (Oxoid CM 1135 ) and incubating at $37^{\circ} \mathrm{C}$ with shaking at $250 \mathrm{rpm}$.

\section{Preparation of Antibacterial Agents}

Fresh antibiotic solutions were prepared for each experiment: ampicillin (dissolved in sterile MilliQ water; Sigma-Aldrich A9518), erythromycin (dissolved in 96\% ethanol; Sigma-Aldrich E6376), gentamicin (dissolved in sterile MilliQ water; SigmaAldrich G3632), norfloxacin (dissolved in sterile MilliQ with 1\% glacial acetic acid; Fluka N9890) vancomycin (dissolved in sterile MilliQ; Sigma-Aldrich V2002) and co-trimoxazole, which is comprised of one part trimethoprim (dissolved in sterile MilliQ with $1 \%$ glacial acetic acid; Sigma-Aldrich 92131) and five parts sulphamethoxazole (dissolved in acetone; Fluka S7507). $\mathrm{H}_{2} \mathrm{O}_{2}$ (30\% in water; Sigma-Aldrich 216763) was diluted to $20 \mathrm{mM}$ in sterile MilliQ water.

\section{Minimal Inhibitory Concentration (MIC) Assay}

Antibiotic MIC values were determined in BHI broth as previously described (Cockerill et al., 2012). In brief, stationary phase cultures of N53-1 and SCV E18 were adjusted to $\mathrm{OD}_{600}=0.2$ and further diluted 1000-fold, corresponding to a $\mathrm{CFU} / \mathrm{ml}$ of $10^{5}$. The adjusted cultures were tested against norfloxacin, ampicillin, gentamicin, and co-trimoxazole (trimethoprim/sulfamethoxazole) using a twofold dilution series of concentrations ranging from 100 to $0.10 \mu \mathrm{g} / \mathrm{ml}$ in 96-well plates (Thermo Scientific 163320) and incubated at $37^{\circ} \mathrm{C}$. MIC for N53-1 and SCV E18 was determined by visual inspection after 24 and $48 \mathrm{~h}$ of incubation at $37^{\circ} \mathrm{C}$, respectively. Two biological replicates were performed.

\section{Killing Kinetic Assays}

To compare the antibiotic and $\mathrm{H}_{2} \mathrm{O}_{2}$ sensitivity of SCV E18 and N53-1, killing kinetics were performed according to Knudsen et al. (2013). Overnight cultures were diluted $10^{6}$-fold in $10 \mathrm{ml}$ BHI broth and incubated into early stationary phase for $16 \mathrm{~h}$ at $37^{\circ} \mathrm{C}$ with shaking at $250 \mathrm{rpm}$. Each culture was then adjusted to an optical density at $600 \mathrm{~nm}\left(\mathrm{OD}_{600}\right)$ of 0.2 in $2 \mathrm{ml} \mathrm{BHI}$ broth and challenged with antibiotic (30X MIC except nitrofurantoin, which was $10 \mathrm{X} \mathrm{MIC})$ at $37^{\circ} \mathrm{C}$. Bacterial counts were performed at $0,4,10,24,48$, and $72 \mathrm{~h}$. The antibiotics and concentrations used were norfloxacin $(100 \mu \mathrm{g} / \mathrm{ml})$, ampicillin (185 $\mu \mathrm{g} / \mathrm{ml})$, erythromycin $(6 \mu \mathrm{g} / \mathrm{ml})$, vancomycin $(47 \mu \mathrm{g} / \mathrm{ml})$, and co-trimoxazole $(95 \mu \mathrm{g} / \mathrm{ml})$. Cultures treated with $\mathrm{H}_{2} \mathrm{O}_{2}$ were grown and adjusted as described above, then treated with $20 \mathrm{mM}$ and incubated under stagnant conditions at $37^{\circ} \mathrm{C}$ for $2 \mathrm{~h}$. Three independent biological replicates were performed for each experiment and the limit of detection was $10^{2} \mathrm{CFU} / \mathrm{ml}$.

\section{Anaerobic Culturing Conditions}

To verify that E18 was sensitive to oxygen, it, along with N531 , were cultured in anaerobic conditions. Oxygen was removed from the media by autoclaving, followed by incubation in a HP0011 anaerobic jar (Oxoid) with an anaerobe gas generation bag (Sigma-Aldrich 68061) for $24 \mathrm{~h}$. Ten microliters of an overnight culture was added to the anaerobic BHI, along with a new anaerobe gas generation bag and incubated on the lab bench for $3 \mathrm{~h}$ to allow for an anaerobic atmosphere to be generated before incubation with shaking. The cultures were grown for $72 \mathrm{~h}$ at $37^{\circ} \mathrm{C}$ with shaking at $200 \mathrm{rpm}$. Two biological replicates were performed.

\section{hemA Complementation}

E18 carries several single nucleotide polymorphisms (SNPs) of which one is in the hemA gene (Kastbjerg et al., 2014). To verify the role of the SNP in hemA in the SCV E18 strain, a hemA complementation strain was constructed. The hemA gene with a $128 \mathrm{bp}$ upstream region including the native promoter was amplified from genomic DNA of N53-1 with the forward primer $5^{\prime}$-AAACTCGAGTCATCCGTTAACTCCTCG and the reverse primer 5'-AAAGAATTCATAGAAGGAGTTGGAATG GA, which contained terminal XhoI and EcoRI sites, respectively, using Phusion High Fidelity DNA Polymerase (NEB, M0530S). The pSOG30222 plasmid (Hain et al., 2008) and hemA amplicon were digested with XhoI and EcoRI, and ligated overnight using a 3:1 insert to vector ratio, then electroporated into $60 \mu \mathrm{l}$ of electrocompetent DH5 $\alpha$ cells using a MicroPulser (BioRad 165-2100). Transformants were selected on Luria-Bertani plates with $100 \mu \mathrm{g} / \mathrm{ml}$ erythromycin and verified by colony PCR and sequencing (GATC Biotech). Plasmids containing the correct pSOG30222::hemA construct, along with an unmodified pSOG30222 control plasmid, were transformed into the SCV E18 strain. Transformants were selected on BHI agar with $5 \mu \mathrm{g} / \mathrm{ml}$ erythromycin and confirmed with colony PCR and sequencing. With the exception of the killing kinetic assay, all complementation experiments were carried out in the presence of $5 \mu \mathrm{g} / \mathrm{ml}$ erythromycin. Colony pictures were taken of each strain with an Olympus BX51 microscope at $40 \times$ magnification.

\section{Macrophage Internalization and Survival Assay}

A murine macrophage cell line, RAW 264.7 (Sigma-Aldrich 91062702), was used to test bacterial internalization and intracellular survival using a protocol adapted from Bateman and Seed (Bateman and Seed, 2012). RAW 264.7 macrophages were grown in Dulbecco's Modified Eagle's Medium - high glucose 
(Sigma-Aldrich D6546), 10\% heat inactivated fetal bovine serum (Sigma-Aldrich F9665) and L-glutamine (Sigma-Aldrich G7513) in $150 \mathrm{cc}$ tissue culture flasks, for $10-15$ passages prior to use. A cell scraper was used to disjoin the adherent cells from the surface. RAW 264.7 cells were seeded into 24 -well plates at a density of $2 \times 10^{6}$ cells $/ \mathrm{ml}$ in RAW 264.7 culture media and then incubated for a total of $48 \mathrm{~h}$ at $37^{\circ} \mathrm{C}$ with $5 \% \mathrm{CO}_{2}$. During the $48 \mathrm{~h}$, one group of RAW 264.7 cells was stimulated for $18 \mathrm{~h}$ with $1 \mathrm{ng} / \mathrm{ml}$ IFN $\gamma$, required for macrophage activation, and a second group was stimulated with $100 \mathrm{nM}$ phorbol 12-myristate 13-acetate (PMA), a known inducer of nicotinamide adenine dinucleotide phosphate (NADPH) oxidase activity (Walloschke et al., 2010) for $2 \mathrm{~h}$, while a third control group was left unstimulated. Prior to infection, IFN $\gamma$ and PMA were removed from the RAW 264.7 containing wells. Overnight cultures of N53-1 and SCV E18 were diluted $10^{6}$-fold in $10 \mathrm{ml}$ of BHI media and grown at $37^{\circ} \mathrm{C}$ for $16 \mathrm{~h}$. L. monocytogenes cultures were adjusted to a density of $2 \times 10^{7} \mathrm{CFU} / \mathrm{ml}$ in $\mathrm{PBS}$ and added to the confluent RAW 264.7 containing wells, achieving a multiplicity of infection (MOI) of 10 . Plates were centrifuged for $5 \mathrm{~min}$ at $1,000 \mathrm{rpm}$ to synchronize the infection and incubated at $37^{\circ} \mathrm{C}$ for $1 \mathrm{~h}$. The plates were then incubated for an additional $2 \mathrm{~h}$ in media containing $100 \mu \mathrm{g} / \mathrm{ml}$ gentamicin, which was reduced to $50 \mu \mathrm{g} / \mathrm{ml}$ gentamicin for the remainder of the incubation. In order to verify that gentamicin did not favor the resistant SCV E18 during the $21 \mathrm{~h}$ incubation step, a control experiment was performed using the same methods as the unactivated macrophage experiment, with the exception that the media with $50 \mu \mathrm{g} / \mathrm{ml}$ gentamicin was substituted with PBS. To enumerate the surviving L. monocytogenes per well, RAW 264.7 cells were washed twice with PBS, followed by lysis with $0.1 \%$ Triton-X 100 (Sigma-Aldrich T8787) and then serial dilutions of the intracellular bacteria were plated on BHI agar plates. Internalization was measured following $3 \mathrm{~h}$ of incubation, while survival was measured after $24 \mathrm{~h}$.

\section{Statistical Analysis}

$\mathrm{CFU} / \mathrm{ml}$ values for each biological replicate were $\log _{10}$ transformed prior to statistical analysis. For the killing kinetic and $\mathrm{O}_{2}$ sensitivity assays, significant differences between the N53-1 and SCV E18 were determined using a paired $t$-test, where significance is equal to $P<0.05$. Significance between N53-1 and SCV E18 for the RAW 264.7 internalization and survival assays was determined using a paired $t$-test $(P<0.05)$ with the $\log _{10}$ transformed CFU/ml values. Significance for each strain between the RAW264.7 treatment groups was calculated using a one-way ANOVA test for the $\log _{10}$ transformed $\mathrm{CFU} / \mathrm{ml}$ values, where $P<0.05$ determined significance. Significance between the two strains within each RAW264.7 treatment group was calculated using a paired $t$-test $(P<0.05)$. The limit of detection for all $\mathrm{CFU} / \mathrm{ml}$ values was $10^{2}$. All data analysis was performed in Excel. Replicates falling below the detection limit were set to 99 for statistical analysis.

\section{RESULTS}

\section{SCV E18 Shows Increased Tolerance to Antibiotics}

The L. monocytogenes SCVs, selected on gentamicin, were resistant to other aminoglycosides (Kastbjerg et al., 2014). To determine if the SCV phenotype conveys resistance to other antibiotics, MIC values of selected antibiotics were tested for both N53-1 and SCV E18, which were found to be the same for the two strains (Table 1).

Because L. monocytogenes SCV grow slower than the wild type (Kastbjerg et al., 2014), and because of the lowered oxidative phosphorylation observed in other SCV organisms, such as S. aureus (Proctor et al., 1998), we hypothesized that L. monocytogenes SCVs would have an increased tolerance to other classes of antibiotics, which was evaluated using time dependent killing experiments. With the exception of erythromycin, the SCV E18 strain survived significantly better for each antibiotic challenge up to $48 \mathrm{~h}$ (Figure 1; ampicillin, $P=0.0007$; co-trimoxazole, $P=0.02$; vancomycin, $P=0.02$ and norfloxacin, $P=0.03$ ), with between one and threefold higher $\log _{10} \mathrm{CFU} / \mathrm{ml}$ as compared to the N53-1. When treated with norfloxacin, both N53-1 and SCV E18 exhibited a biphasic killing curve with a rapid decline to approximately three and five $\log _{10}$ $\mathrm{CFU} / \mathrm{ml}$, respectively, which remained stable throughout the $72 \mathrm{~h}$ experiment (Figure 1A). Ampicillin has a delayed bactericidal effect on L. monocytogenes (Winslow et al., 1983), which was also observed with SCV E18, as is evidenced by the roughly three-fold $\log _{10}$ reduction in CFU/ml over the $48-72 \mathrm{~h}$ time points (Figure 1B). However, this delayed bactericidal effect of ampicillin was not observed for N53-1 over the course of the

TABLE 1 | Minimum Inhibitory Concentration (MIC) values for Listeria monocytogenes wild type strain N53-1 strain and SCV E18 strain.

\begin{tabular}{|c|c|c|c|c|c|c|}
\hline \multirow[b]{2}{*}{ Strain } & \multicolumn{6}{|c|}{$\mathrm{MIC} \mu \mathrm{g} / \mathrm{ml}$} \\
\hline & Ampicillin & Co-trimoxazole & Norfloxacin & Erythromycin & Vancomycin & Gentamicin \\
\hline N53-1 & 3.13 & 0.78 & 3.13 & 0.20 & 1.56 & 0.40 \\
\hline E18 & 3.13 & 0.78 & 3.13 & 0.20 & 1.56 & 12.50 \\
\hline $\begin{array}{l}\text { E18 } \\
\text { pSOG30222::hemA }\end{array}$ & 1.56 & 0.78 & 3.13 & - & 1.56 & 0.40 \\
\hline E18 pSOG30222 & 1.56 & 0.78 & 3.13 & - & 1.56 & 12.50 \\
\hline
\end{tabular}

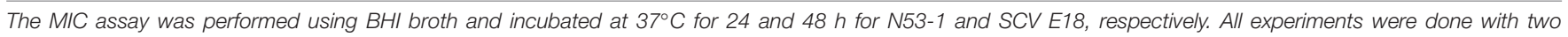
independent replicates. - , not tested. 


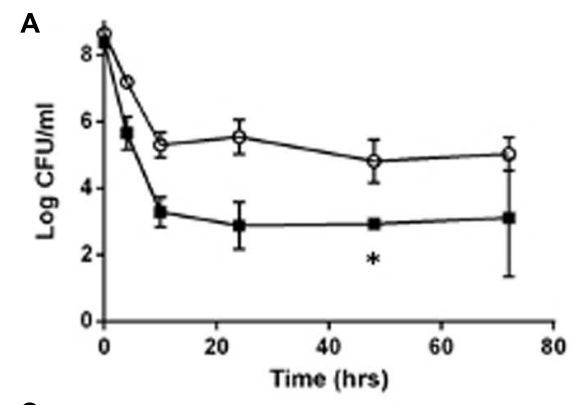

C

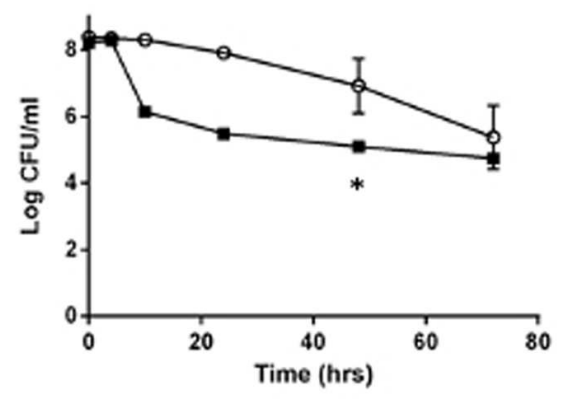

E

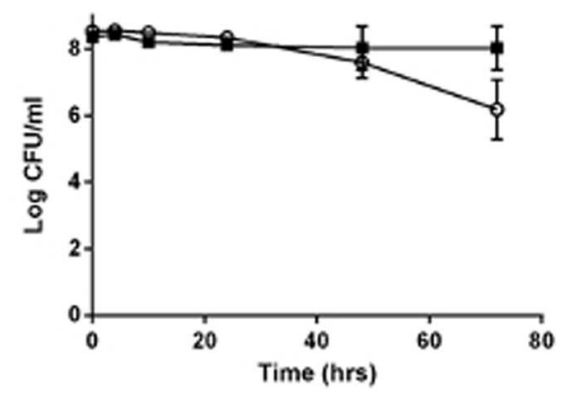

B

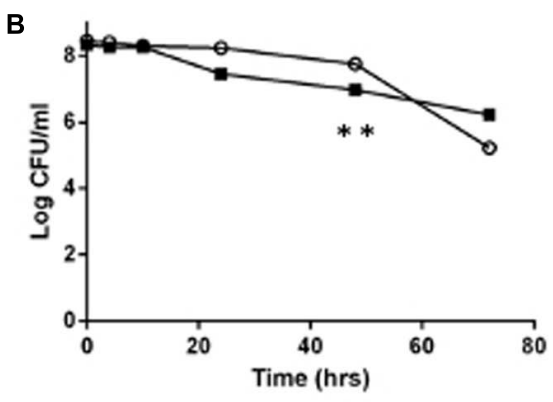

D

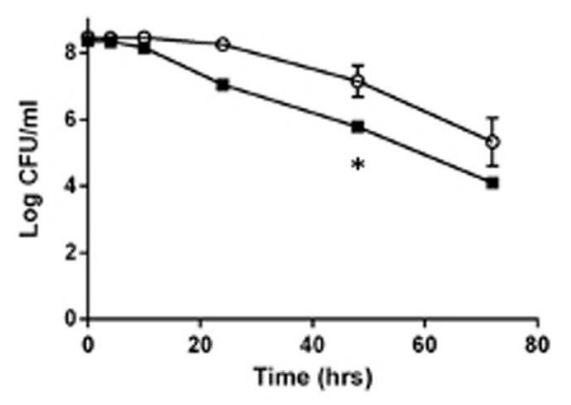

FIGURE 1 | Killing of Listeria monocytogenes N53-1 ( $\square$ ) and SCV E18 (O) over the course of $72 \mathrm{~h}$ under shaking conditions with (A) norfloxacin, (B) ampicillin, (C) co-trimoxazole, (D) vancomycin, and (E) erythromycin. Error bars represent standard deviation of the mean for three biological replicates. Significance at the 48-h time point was determined using a paired $t$-test $\left({ }^{*} p<0.05\right.$ and $\left.{ }^{* *} p<0.005\right)$.

$72 \mathrm{~h}$ killing kinetic (Figure 1B). By the end of the $72 \mathrm{~h}$ treatment with co-trimoxazole, SCV E18 decreased in a linear manner to a comparable level with N53-1 $\left(2.3 \times 10^{5}\right.$ and $5.6 \times 10^{4}$ $\mathrm{CFU} / \mathrm{ml}$, respectively; $P=0.45$; Figure $1 \mathrm{C})$. Vancomycin had an initial bacteriostatic effect on both strains, followed by linear killing, however, the bactericidal effect began earlier for N53-1 $(T=10)$, while the SCV E18 strain resisted killing until the $24 \mathrm{~h}$ time point (Figure 1D). Erythromycin was bacteriostatic on both strains up to the $48 \mathrm{~h}$ time point, however, bacterial counts for SCV E18 decreased after $48 \mathrm{~h}$, while the N53-1 remained stable (Figure 1E).

While the SCV phenotype in L. monocytogenes does not confer notable resistance to antibiotics besides the aminoglycosides, they survived significantly better under high antibiotic concentrations for up to $48 \mathrm{~h}$ under ampicillin or co-trimoxazole, and up to $72 \mathrm{~h}$ when exposed to norfloxacin or vancomycin. In contrast, by $72 \mathrm{~h}$ of exposure to erythromycin or ampicillin, the SCV has fewer $\mathrm{CFU} / \mathrm{ml}$ than the wild type.

\section{Oxygen Sensitivity, But $\mathrm{H}_{2} \mathrm{O}_{2}$ Resistance, of the SCV E18}

When grown under shaking conditions, SCV E18 grows to a maximum density of approximately $1 \times 10^{9} \mathrm{CFU} / \mathrm{ml}$ after $12 \mathrm{~h}$, where after the CFU/ml decreases rapidly, eventually dropping below the limit of detection after $24 \mathrm{~h}$ (Kastbjerg et al., 2014). Here, we grew cultures of the SCV E18, along with the N53-1, anaerobically to test if the observed decrease in CFU/ml is caused by exposure to oxygen. When grown anaerobically, cultures of the SCV E18 grow to higher densities $\left(8.1 \times 10^{8} \mathrm{CFU} / \mathrm{ml}\right)$ after $72 \mathrm{~h}$, than the N53-1 strain $\left(2.5 \times 10^{8} \mathrm{CFU} / \mathrm{ml} ; P=0.002\right)$ (Table 2), suggesting that oxygen is toxic to the SCV E18 strain. Under anaerobic growth conditions the SCV E18 strain retained the pin point colony morphology when plated, whereas the N53-1 strain exhibited variable colony size.

To test if the oxygen sensitivity was related to a general deficiency in the oxidative stress response, such as the absence of catalase in the SCV E18, we treated with $20 \mathrm{mM} \mathrm{H}_{2} \mathrm{O}_{2}$ 
TABLE 2 | Oxidative stress sensitivity of L. monocytogenes N53-1 and SCV E18 as measured by aerobic and anaerobic growth, and exposure to 20 mM $\mathrm{H}_{2} \mathrm{O}_{2}$ for $2 \mathrm{~h}$.

\begin{tabular}{|c|c|c|c|c|}
\hline & \multicolumn{4}{|c|}{ Bacterial count $\left(\log _{10} \mathrm{CFU} / \mathrm{ml}\right)$} \\
\hline & \multicolumn{2}{|c|}{ Growth in $\mathrm{BHI}$ for $72 \mathrm{~h}$} & \multicolumn{2}{|c|}{ Survival in $20 \mathrm{mM} \mathrm{H} \mathrm{H}_{2}$} \\
\hline & Aerobic & Anaerobic & $\mathbf{O h}$ & $2 \mathrm{~h}$ \\
\hline N53-1 & $9.4 \pm .01$ & $8.4 \pm 0.03$ & $7.2 \pm 0.04$ & $3.7 \pm 0.09$ \\
\hline E18 & $\mathrm{BD}$ & $8.9 \pm 0.02$ & $7.2 \pm 0.13$ & $6.3 \pm 1.02$ \\
\hline E18 pSOG30222::hemA & $8.1 \pm 0.09$ & - & $8.0 \pm 0.22$ & $5.2 \pm 0.17$ \\
\hline E18 pSOG30222 & $\mathrm{BD}$ & - & $8.0 \pm 0.06$ & $6.5 \pm 0.41$ \\
\hline
\end{tabular}

All experiments are $\log _{10} \mathrm{CFU} / \mathrm{ml}$ mean values of three biological replicates \pm standard deviation. BD, below the limit of detection; -, not tested.

for 2 h. Surprisingly, the SCV E18 survived significantly better $(P=0.008)$ than the wild type, with the number of colony forming bacteria following treatment being 33\% of the inoculum, whereas the N53-1 strain survived at a rate of $0.03 \%$ (Table 2). Thus, similar to the increased tolerance toward antibiotics, and regardless of the oxygen sensitivity and lack of catalase (Kastbjerg et al., 2014), the SCV E18 strain exhibits a greater ability to resist killing by $\mathrm{H}_{2} \mathrm{O}_{2}$ over the wild type.

\section{hemA Complementation Restores Wild Type Phenotype of SCV E18}

By genome sequencing and analyses, we previously demonstrated that all of the L. monocytogenes SCVs had several mutations, including a mutation in the hemA gene (Kastbjerg et al., 2014). To determine if the tested aspects of the SCV phenotype were due to the SNP in the hemA gene, a wild type hemA complemented strain of E18 was constructed. This complemented strain regained the large colony phenotype (Figure 2A), had restored gentamicin sensitivity identical to the N53-1 strain (Table 1), regained sensitivity to $\mathrm{H}_{2} \mathrm{O}_{2}$ and lost its sensitivity to oxygen, as demonstrated by the presence of viable colonies after culturing for $72 \mathrm{~h}$ (Table 2). Furthermore, the level of antibiotic tolerance was evaluated with a time dependent killing experiment using norfloxacin. The killing curve of the complemented hemA strain was significantly different from the empty vector control $(P=0.0000046)$, and was similar to the killing of N53-1 strain, while the empty vector control showed a very similar curve to its non-transformed counterpart SCV E18 (Figure 2B).

\section{SCV E18 Is Less Affected by Macrophage Status Than Wild Type N53-1}

We investigated the survival ability of the wild type and the SCV E18 in the IFN $\gamma$ activated and un-stimulated macrophage model. Additionally, due to the increased tolerance of SCV E18 toward $\mathrm{H}_{2} \mathrm{O}_{2}$, we used macrophages stimulated with PMA, a known activator of the NADPH oxidase pathway. Three hours following infection of the un-stimulated macrophage there was a high degree of internalization and intracellular growth of N53-1 and SCV E18, with $7.6 \log _{10} \mathrm{CFU} / \mathrm{ml}$ and $7.5 \log _{10} \mathrm{CFU} / \mathrm{ml}$ recovered, respectively, a difference that was not statistically significant $(P=0.77)$. These internalization findings were similar for the other two treatment groups (Figure 3A). By 24 h of incubation in

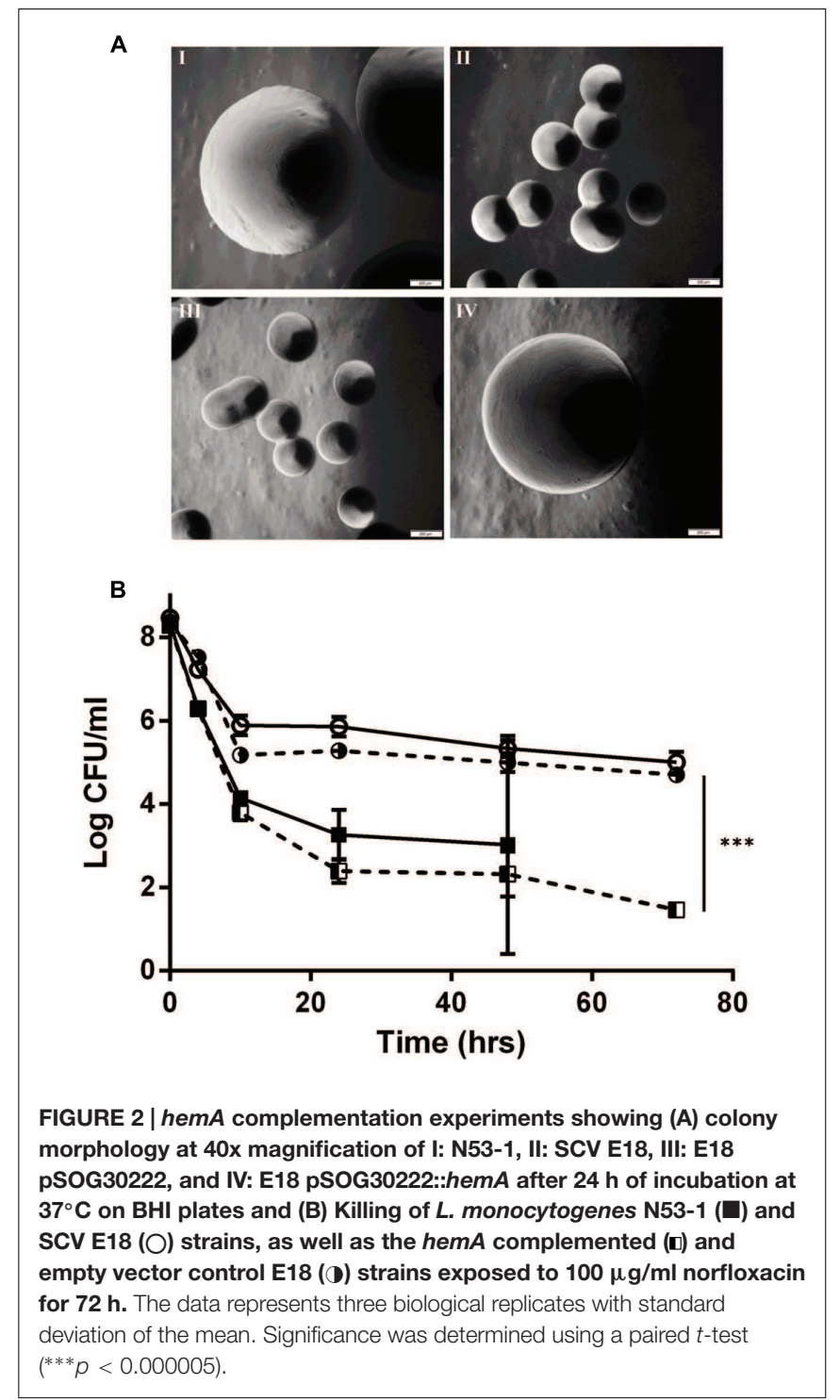

the un-stimulated macrophage, bacterial counts were drastically reduced for both strains, although N53-1 survived significantly better $(P=0.006)$ with $3.6 \log _{10} \mathrm{CFU} / \mathrm{ml}$ of the N53-1 and 2.3 $\log _{10} \mathrm{CFU} / \mathrm{ml}$ for SCV E18 (Figure 3B). When macrophages 

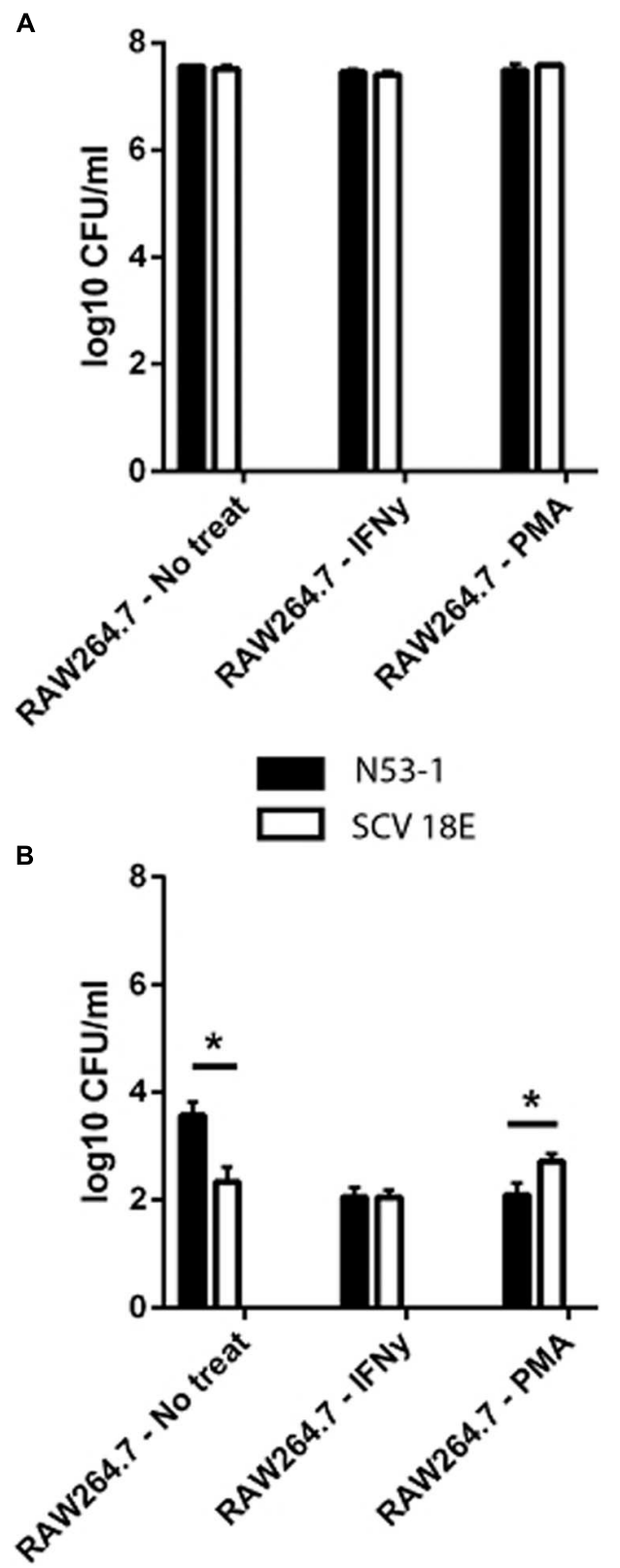

FIGURE 3 | Intracellular (A) internalization and (B) survival of L. monocytogenes N53-1 and SCV E18 in RAW264.7 cells treated with IFN $\gamma$, PMA and no treatment using a multiplicity of infection (MOI) of 10. Bacterial counts for the internalization assay were determined after $2 \mathrm{~h}$ of $100 \mu \mathrm{g} / \mathrm{ml}$ gentamicin exposure. Survival was determined after 21 additional hours of incubation with $50 \mu \mathrm{g} / \mathrm{ml}$ gentamicin. Values are displayed as the $\log _{10}$ transformed mean of three biological replicates with standard deviation. Significance was determined using a paired $t$-test $\left({ }^{*} p<0.05\right)$.

were activated with IFN $\gamma$, the survival difference between strains vanished, as the number of recovered N53-1 bacteria at $24 \mathrm{~h}$ was reduced to $2.06 \log _{10} \mathrm{CFU} / \mathrm{ml}$, while the SCV E18 burden was almost unchanged by IFN $\gamma$ activation $\left(2.15 \log _{10} \mathrm{CFU} / \mathrm{ml}\right)$ (Figure 3B). Increased reactive oxygen species (ROS) production, via PMA activation of the NADPH oxidase pathway, resulted in a slight, but significantly higher bacterial burden of macrophages infected with SCV E18 (2.7 $\left.\log _{10} \mathrm{CFU} / \mathrm{ml}\right)$, than N53-1 $\left(2.1 \log _{10}\right.$ $\mathrm{CFU} / \mathrm{ml} ; P=0.02$ ) (Figure 3B). While activation of macrophages with IFN $\gamma$ or stimulation with PMA significantly reduced the intracellular bacterial load of N53-1 $(P=0.01)$, the SCV E18 was not sensitive to either treatment and remained unchanged across groups $(P=0.17)$.

To summarize, macrophages were equally poor at controlling the bacterial burden of both N53-1 and SCV E18 in the short term $(T=3 \mathrm{~h})$, however, by $24 \mathrm{~h}$ the $\mathrm{CFU} / \mathrm{ml}$ for all intracellular bacteria had been severely reduced. While survival in the unactivated macrophage was attenuated for SCV E18, as compared to N53-1, it was unaffected by the status of the macrophage unlike N53-1, which was significantly reduced in CFU/ml upon macrophage activation with IFN $\gamma$ or stimulation with PMA.

\section{DISCUSSION}

The Small Colony Variant (SCV) has been described for decades in several bacteria such as $S$. aureus, E. coli and $P$. aeruginosa (Proctor et al., 2006). However, they were only recently identified in L. monocytogenes and, with the exception of aminoglycoside resistance (Kastbjerg et al., 2014), their importance with respect to the treatment of listeriosis has not been investigated. This study is the first to demonstrate that the SCV phenotype of L. monocytogenes caused by a single point mutation in the hem $A$ gene has the potential to complicate treatment by causing an increase in tolerance toward most of the clinically relevant antibiotics.

As shown previously (Kastbjerg et al., 2014), SCV E18 exhibited resistance to the aminoglycoside gentamicin, a defining trait of the electron-transport deficient SCV (Proctor et al., 2006). This is particularly relevant for listeriosis, as gentamicin is the most common secondary antibiotic used for treatment (Temple and Nahata, 2000). While the SCV E18 showed no increase in MIC for the other antibiotics tested, indicating a lack of acquired resistance, it was nonetheless able to survive lethal concentrations of norfloxacin, ampicillin (up to $48 \mathrm{~h}$ ), co-trimoxazole, and vancomycin significantly better than the wild type, which taken together, are the definition of antibiotic tolerance (Dimitrijovski et al., 2015). The increase in tolerance toward vancomycin, second generation fluoroquinolones (e.g., norfloxacin) and betalactams (e.g., ampicillin) has been observed with $S$. aureus SCVs and is presumably the result of their slow growing SCV phenotype (Chuard et al., 1997; Garcia et al., 2012; Lechner et al., 2012). Antibiotic tolerance poses a unique clinical dilemma, as any testing on isolates relying solely on MIC values could obscure the true antimicrobial susceptibility of the SCV and lead to sub-optimal treatment strategies.

In contrast to the other antibiotics, SCV E18 did not survive better under erythromycin pressure at any time point during the experiment and, as with ampicillin, became more sensitive after $48 \mathrm{~h}$ of killing. This could indicate that whatever protective effect the SCV phenotype contributes toward other antibiotics does not apply to the macrolide class, which target protein synthesis. Interestingly, S. aureus SCVs were not isolated from cystic fibrosis 
patients treated exclusively with the macrolide azithromycin, whereas when treated with other antibiotics, SCVs were observed in $46 \%$ of patients colonized with S. aureus (Kahl et al., 2003; Green et al., 2011). Although the SCV L. monocytogenes E18 tolerates ampicillin better than the wild type N53-1 in the short term, the sensitivity toward extended exposure (as evidenced by the pronounced decrease in bacterial count following $48 \mathrm{~h}$ of treatment) and the fact that long-term (between 2 and 8 weeks) ampicillin therapy is the primary treatment of choice for listeriosis (Temple and Nahata, 2000), could explain why the SCV phenotype has, to our knowledge, not been observed in in clinical cases. However, it is also possible that current screening procedures in the clinical laboratory are inadequate, leading the SCV sub-population to go unnoticed (Proctor et al., 2006). The slower growth of SCV E18 would likely cause it to be overshadowed in a mixed culture containing the wild type. Furthermore, the altered sugar metabolism and lack of catalase observed in SCV E18 (Kastbjerg et al., 2014), as well as the fact that some of the media used to grow L. monocytogenes contain the compounds SCVs are auxotrophic for, would complicate proper identification of any SCV L. monocytogenes clinical isolates.

We were expecting the SCV E18 to be more sensitive to $\mathrm{H}_{2} \mathrm{O}_{2}$ because it does not produce catalase, however, it was able to better withstand $\mathrm{H}_{2} \mathrm{O}_{2}$ than the wild type. As $\mathrm{H}_{2} \mathrm{O}_{2}$ reacts with the heme cofactor of proteins (Nagababu and Rifkind, 1998), our observation may simply be the result of fewer targets in the heme deficient SCV E18. Alternatively, tolerance toward $\mathrm{H}_{2} \mathrm{O}_{2}$ may be due to a change in the metabolism of the SCV, as suggested by Painter et al. (2015). In addition to showing that heme auxotrophic SCVs of $S$. aureus were more tolerant of $\mathrm{H}_{2} \mathrm{O}_{2}$, they added 4-hydroxy-2-heptylquinoline- $N$-oxide (HQNO), which can induce the SCV phenotype by blocking the electron transport chain of Gram-positive bacteria, to wild type $S$. aureus either hours or minutes before exposure to lethal levels of $\mathrm{H}_{2} \mathrm{O}_{2}$. Those bacteria grown for hours in the presence of HQNO survived the $\mathrm{H}_{2} \mathrm{O}_{2}$ exposure significantly better than the wild type, whereas those that received the HQNO only minutes before did not. This demonstrates that the tolerance is not simply the result of reduced electron transport, but as the authors suggest, a global physiological change caused by the loss of the electron transport chain (e.g., a switch to anaerobic metabolism), which could also explain our similar observation in L. monocytogenes.

In addition to the typical characteristics of the SCV, L. monocytogenes SCVs exhibit a unique sensitivity to growth under shaking conditions as they enter the stationary phase (Kastbjerg et al., 2014). Here, we demonstrate that this results from the presence of oxygen in the media. The addition of catalase, which converts $\mathrm{H}_{2} \mathrm{O}_{2}$ to water and oxygen, or heme (necessary for the biosynthesis of catalase) to the growth media abolished this effect, suggesting that the oxygen sensitivity is due to the inability of the heme-deficient SCV mutant to produce catalase (Kastbjerg et al., 2014). However, to our knowledge, this oxygen sensitivity has not been observed in heme-deficient SCVs of other catalase-dependent species (Roggenkamp et al., 1998; Zahra et al., 2013), and this fails to explain why the associated toxicity only manifests in the stationary phase. Few explanations seem to coincide with the observed increased tolerance toward
$\mathrm{H}_{2} \mathrm{O}_{2}$ and extended viability of SCV E18 cells when treated with antibiotics, however, we believe this is an important observation that warrants further research.

When the wild type and SCV E18 were inoculated with RAW264.7 murine macrophages over the course of $3 \mathrm{~h}$, we observed no difference between the internalization of the two strains. However, prolonged incubation of the infected macrophages revealed differences between the fates of each strain depending on the activation status of the macrophages. N53-1 survived better than SCV E18 in the naïve macrophages, however, this difference disappeared once macrophages were activated with IFN $\gamma$ where the wild type was reduced to the same CFU/ml as the mutant. The former observation is likely explained by the reduced expression of the pore-forming toxin Listeriolysin $\mathrm{O}$ (LLO) (Kastbjerg et al., 2014) in SCV E18, which has been shown to be produced in murine macrophages (Moors et al., 1999) and is necessary for L. monocytogenes to escape from the phagosome (Bielecki et al., 1990). The latter observation corresponds with studies showing that IFN $\gamma$-activated macrophages are able to block the escape of L. monocytogenes (Portnoy et al., 1989) by a process thought to be mediated by ROS inactivation of the LLO toxin (Myers et al., 2003). This suggests that macrophages need not first be activated by IFN $\gamma$ in order to control L. monocytogenes SCVs, which contrasts to SCVs from other species, and could explain the lack of observed clinical L. monocytogenes SCVs. On the other hand, LLO is the most immunogenic antigen for the $\mathrm{T}$ cell response, which mediates the ultimate removal and adaptive immunity to L. monocytogenes (Vijh and Pamer, 1997), thus a reduction in LLO expression may lead to a weaker adaptive immune response to SCV E18 and could explain the observation that mutants incapable of producing LLO persist for significantly longer in the bone marrow of infected mice (Hardy et al., 2009). Furthermore, the PMA stimulated macrophage showed that SCV E18 is significantly better at surviving the bactericidal effects of ROS within the phagosome, which is consistent with its observed increase in $\mathrm{H}_{2} \mathrm{O}_{2}$ tolerance, and could have implications on the fate of SCV L. monocytogenes in neutrophils that rely predominantly on NADPH oxidase for their bactericidal activity (Segal, 2005).

Our complementation experiment showed that this phenotype can arise through a single base-pair substitution in the hemA gene, and given the prevalence of verified SCV selection pressures L. monocytogenes faces both in the environment and during treatment (e.g., triclosan, gentamicin and co-trimoxazole), the probability of exposure to SCV L. monocytogenes seems high. Therefore, it is surprising that no clinical cases of SCV associated listeriosis have been reported. Perhaps the long treatment with ampicillin is enough to eradicate the SCV, or the immune system is better able to control SCV L. monocytogenes, as indicated by our findings with non-IFN $\gamma$ activated RAW264.7 murine macrophages. However, it seems equally likely that this elusive phenotype has gone unnoticed due to a lack of adequate screening procedures in clinical laboratories.

Much like persister cells, SCVs have also been shown to revert to the wildtype phenotype. When clinical and laboratory strains of $S$. aureus were cyclically grown with and without gentamicin, an identical proportion of SCVs upon each 
exposure to the antibiotic was observed (Massey et al., 2001). Drenkard and Ausubel (2002) found the same phenotypic switching in $P$. aeruginosa, and also identified a protein that modulates the switch between the wild type and SCV phenotypes. This phenotypic heterogeneity, often referred to as a bet-hedging strategy, allows a portion of the bacterial population to survive a stress (e.g., antibiotic exposure or the phagosome), without giving up any fitness advantages associated with mutation. It is plausible that the majority of SCVs in vivo are the result of phenotypic switching, and although, we observed no reversion with SCV E18, this mutant and others like it could represent a small subset of SCVs locked in the on position. This would add another treatment challenge, as the bacterial population as a whole would be protected from antibiotics, while simultaneously capable of full virulence (Tuchscherr et al., 2011). Reports of SCVs from a variety of bacterial species being linked to persistent and chronic infections are numerous (Proctor et al., 1995; von Eiff et al., 1997; Roggenkamp et al., 1998; Seifert et al., 1999; Häussler et al., 2003), and while uncommon, cases of recurrent listeriosis have been reported and the cause of these treatment failures were not determined (McLauchlin et al., 1991; Sauders et al., 2001; Kleemann et al., 2009).

Although L. monocytogenes SCVs may be unique in that macrophages are able to control them without first becoming activated by IFN $\gamma$, they may yet be able to better survive other aspects of the immune system, and the presence of an easily generated, multi-antibiotic tolerant sub-population

\section{REFERENCES}

Balaban, N. Q., Merrin, J., Chait, R., Kowalik, L., and Leibler, S. (2004). Bacterial persistence as a phenotypic switch. Science 305, 1622-1625. doi: 10.1126/science. 1099390

Balwit, J. M., van Langevelde, P., Vann, J. M., and Proctor, R. A. (1994). Gentamicin-resistant menadione and hemin auxotrophic Staphylococcus aureus persist within cultured endothelial cells. J. Infect. Dis. 170, 1033-1037. doi: 10.1093/infdis/170.4.1033

Bateman, S. L., and Seed, P. C. (2012). Epigenetic regulation of the nitrosative stress response and intracellular macrophage survival by extraintestinal pathogenic Escherichia coli. Mol. Microbiol. 83, 908-925. doi: 10.1111/j.13652958.2012.07977.x

Besier, S., Ludwig, A., Ohlsen, K., Brade, V., and Wichelhaus, T. A. (2007). Molecular analysis of the thymidine-auxotrophic small colony variant phenotype of Staphylococcus aureus. Int. J. Med. Microbiol. 297, 217-225. doi: 10.1016/j.ijmm.2007.02.003

Bielecki, J., Youngman, P., Connelly, P., and Portnoy, D. A. (1990). Bacillus subtilis expressing a haemolysin gene from Listeria monocytogenes can grow in mammalian cells. Nature 345, 175-176. doi: 10.1038/345175a0

Chatterjee, I., Kriegeskorte, A., Fischer, A., Deiwick, S., Theimann, N., Proctor, R. A., et al. (2008). In vivo mutations of thymidylate synthase (encoded by thyA) are responsible for thymidine dependency in clinical small-colony variants of Staphylococcus aureus. J. Bacteriol. 190, 834-842. doi: 10.1128/JB. 00912-07

Christensen, E. G., Gram, L., and Kastbjerg, V. G. (2011). Sublethal triclosan exposure decreases susceptibility to gentamicin and other aminoglycosides in Listeria monocytogenes. Antimicrob. Agents Chemother. 55, 4064-4071. doi: 10.1128/AAC.00460-11

Chuard, C., Vaudaux, P. E., Proctor, R. A., and Lew, D. P. (1997). Decreased susceptibility to antibiotic killing of a stable small colony variant of Staphylococcus aureus in fluid phase and on fibronectin-coated surfaces. J. Antimicrob. Chemother. 39, 603-608. doi: 10.1093/jac/39.5.603 is nonetheless a cause for concern. Awareness and adequate screening for SCVs in clinical isolates, such as extended incubation times and inclusion of isolates with non-standard sugar metabolism or those lacking catalase activity, would also help to determine the role (if any) this phenotype plays in human infection with $L$. monocytogenes, and we believe the evidence presented here warrants such measures being employed in clinical laboratories.

\section{AUTHOR CONTRIBUTIONS}

Conceived and designed the experiments: TC, LG, and GK. Performed the experiments: TC. Analyzed the data: TC, LG, and GK. Wrote the manuscript: TC, LG, and GK.

\section{FUNDING}

This work was supported by a Ph.D. grant from the Department of Systems Biology, Technical University of Denmark.

\section{ACKNOWLEDGMENT}

The authors would like to thank all members of the Gram Lab for their support and feedback.

Cockerill, F. R.,Wiker, M. A., Alder, J., Dudley, M. N., Eliopoulos, G. M., Ferraro, M. J., et al. (2012). Methods for Dilution Antimicrobial Susceptibility Testing for Bacteria that Grow Aerobically. Approved Standard - 9th Edn, Vol. 32. Wayne, PA: Clinical and Laboratory Standards Institute, 18-20.

Dimitrijovski, B., Jensen, S. O., Espedido, B. A., and van Hal, S. J. (2015). "Tolerance" of Misused Terminology? Enforcing Standardized Phenotypic Definitions. MBio 6:e00446-15. doi: 10.1128/mBio.00446-15

Drenkard, E., and Ausubel, F. M. (2002). Pseudomonas biofilm formation and antibiotic resistance are linked to phenotypic variation. Nature 416, 740-743. doi: $10.1038 / 416740 \mathrm{a}$

Edwards, A. M. (2012). Phenotype switching is a natural consequence of Staphylococcus aureus replication. J. Bacteriol. 194, 5404-5412. doi: 10.1128/JB.00948-12

Farber, J. M., and Peterkin, P. I. (1991). Listeria monocytogenes, a food-borne pathogen. Microbiol. Rev. 55, 476-511.

Fridman, O., Goldberg, A., Ronin, I., Shoresh, N., and Balaban, N. Q. (2014). Optimization of lag time underlies antibiotic tolerance in evolved bacterial populations. Nature 513, 418-421. doi: 10.1038/nature13469

Garcia, L. G., Lemaire, S., Kahl, B. C., Becker, K., Proctor, R. A., Denis, O., et al. (2012). Pharmacodynamic evaluation of the activity of antibiotics against hemin- and menadione-dependent small-colony variants of Staphylococcus aureus in models of extracellular (broth) and intracellular (THP-1 monocytes) infections. Antimicrob. Agents Chemother. 56, 3700-3711. doi: 10.1128/AAC.00285-12

Garcia, L. G., Lemaire, S., Kahl, B. C., Becker, K., Proctor, R. A., Denis, O., et al. (2013). Antibiotic activity against small-colony variants of Staphylococcus aureus: review of in vitro, animal and clinical data. J. Antimicrob. Chemother. 68, 1455-1464. doi: 10.1093/jac/dkt072

Green, N., Burns, J. L., Mayer-Hamblett, N., Kloster, M., Lands, L. C., Anstead, M., et al. (2011). Lack of association of small-colony-variant Staphylococcus aureus strains with long-term use of azithromycin in patients with cystic fibrosis. J. Clin. Microbiol. 49, 2772-2773. doi: 10.1128/JCM. 00835-11 
Hain, T., Hossain, H., Chatterjee, S. S., Machata, S., Volk, U., Wagner, S., et al. (2008). Temporal transcriptomic analysis of the Listeria monocytogenes EGD-e sigmaB regulon. BMC Microbiol. 8:20. doi: 10.1186/14712180-8-20

Hardy, J., Chu, P., and Contag, C. H. (2009). Foci of Listeria monocytogenes persist in the bone marrow. Dis. Mode. Mech. 2, 39-46. doi: 10.1242/dmm. 000836

Häussler, S., Lehmann, C., Breselge, C., Rohde, M., Classen, M., Tümmler, B., et al. (2003). Fatal outcome of lung transplantation in cystic fibrosis patients due to small-colony variants of the Burkholderia cepacia complex. Eur. J. Clin. Microbiol. Infect. Dis. 22, 249-253.

Haussler, S., Tummler, B., Weissbrodt, H., Rohde, M., and Steinmetz, I. (1999). Small-colony variants of Pseudomonas aeruginosa in cystic fibrosis. Clin. Infect. Dis. 29, 621-625. doi: 10.1086/598644

Helaine, S., Cheverton, A. M., Watson, K. G., Faure, L. M., Matthews, S. A., and Holden, D. W. (2014). Internalization of Salmonella by macrophages induces formation of nonreplicating persisters. Science 343, 204-208. doi: 10.1126/science. 1244705

Kahl, B. C., Becker, K., and Löffler, B. (2016). Clinical significance and pathogenesis of Staphylococcal small colony variants in persistent infections. Clin. Microbiol. Rev. 29, 401-427. doi: 10.1128/CMR.00069-15

Kahl, B. C., Duebbers, A., Lubritz, G., Haeberle, J., Koch, H. G., Ritzerfeld, B. et al. (2003). Population dynamics of persistent Staphylococcus aureus isolated from the airways of cystic fibrosis patients during a 6-year prospective study. J. Clin. Microbiol. 41, 4424-4427. doi: 10.1128/JCM.41.9.44244427.2003

Kastbjerg, V. G., Hein-Kristensen, L., and Gram, L. (2014). Triclosaninduced aminoglycoside-tolerant Listeria monocytogenes isolates can appear as small-colony variants. Antimicrob. Agents Chemother. 58, 3124-3132. doi: 10.1128/AAC.02266-13

Kleemann, P., Domann, E., Chakraborty, T., Bernstein, I., and Lohoff, M. (2009). Chronic prosthetic joint infection caused by Listeria monocytogenes. J. Med. Microbiol. 58, 138-141. doi: 10.1099/jmm.0.004234-0

Knudsen, G. M., Ng, Y., and Gram, L. (2013). Survival of bactericidal antibiotic treatment by a persister subpopulation of Listeria monocytogenes. Appl. Environ. Microbiol. 79, 7390-7397. doi: 10.1128/AEM.02184-13

Lechner, S., Lewis, K., and Bertram, R. (2012). Staphylococcus aureus persisters tolerant to bactericidal antibiotics. J. Mol. Microbiol. Biotechnol. 22, 235-244. doi: $10.1159 / 000342449$

Lewis, K. (2010). Persister cells. Annu. Rev. Microbiol. 64, 357-372. doi: 10.1146/annurev.micro.112408.134306

Maisonneuve, E., and Gerdes, K. (2014). Molecular mechanisms underlying bacterial persisters. Cell 157, 539-548. doi: 10.1016/j.cell.2014.02.050

Massey, R. C., Buckling, A., and Peacock, S. J. (2001). Phenotypic switching of antibiotic resistance circumvents permanent costs in Staphylococcus aureus. Curr. Biol. 11, 1810-1814. doi: 10.1016/S0960-9822(01)00507-3

McLauchlin, J., Audurier, A., and Taylor, A. G. (1991). Treatment failure and recurrent human listeriosis. J. Antimicrob. Chemother. 27, 851-857. doi: $10.1093 / \mathrm{jac} / 27.6 .851$

Mitjà, O., Pigrau, C., Ruiz, I., Vidal, X., Almirante, B., Planes, A.-M., et al. (2009). Predictors of mortality and impact of aminoglycosides on outcome in listeriosis in a retrospective cohort study. J. Antimicrob. Chemother. 64, 416-423. doi: $10.1093 / \mathrm{jac} / \mathrm{dkp} 180$

Moors, M. A., Levitt, B., Youngman, P., and Portnoy, D. A. (1999). Expression of listeriolysin $\mathrm{O}$ and ActA by intracellular and extracellular Listeria monocytogenes. Infect. Immun. 67, 131-139.

Morvan, A., Moubareck, C., Leclercq, A., Hervé-Bazin, M., Bremont, S., Lecuit, M., et al. (2010). Antimicrobial resistance of Listeria monocytogenes strains isolated from humans in France. Antimicrob. Agents Chemother. 54, 2728-2731. doi: 10.1128/AAC.01557-09

Myers, J. T., Tsang, A. W., and Swanson, J. A. (2003). Localized reactive oxygen and nitrogen intermediates inhibit escape of Listeria monocytogenes from vacuoles in activated macrophages. J. Immunol. 171, 5447-5453. doi: 10.4049/jimmunol.171.10.5447

Nagababu, E., and Rifkind, J. M. (1998). Formation of fluorescent heme degradation products during the oxidation of hemoglobin by hydrogen peroxide. Biochem. Biophys. Res. Commun. 247, 592-596. doi: $10.1006 /$ bbrc. 1998.8846
Painter, K. L., Strange, E., Parkhill, J., Bamford, K. B., Armstrong-James, D., and Edwards, A. M. (2015). Staphylococcus aureus adapts to oxidative stress by producing H2O2-resistant small-colony variants via the SOS response. Infect. Immun. 83, 1830-1844. doi: 10.1128/IAI.03016-14

Portnoy, D. A., Auerbuch, V., and Glomski, I. J. (2002). The cell biology of Listeria monocytogenes infection: the intersection of bacterial pathogenesis and cell-mediated immunity. J. Cell Biol. 158, 409-414. doi: 10.1083/jcb.200 205009

Portnoy, D. A., Schreiber, R. D., Connelly, P., and Tilney, L. G. (1989). Gamma interferon limits access of Listeria monocytogenes to the macrophage cytoplasm. J. Exp. Med. 170, 2141-2146. doi: 10.1084/jem.170.6.2141

Proctor, R. A., Kahl, B., Von Eiff, C., Vaudaux, P. E., Lew, D. P., and Peters, G. (1998). Staphylococcal small colony variants have novel mechanisms for antibiotic resistance. Clin. Infect. Dis. 27, 68-74. doi: 10.1086/ 514906

Proctor, R. A., Kriegeskorte, A., Kahl, B. C., Becker, K., Löffler, B., and Peters, G. (2014). Staphylococcus aureus Small Colony Variants (SCVs): a road map for the metabolic pathways involved in persistent infections. Front. Cell. Infect. Microbiol. 4:99. doi: 10.3389/fcimb.2014.00099

Proctor, R. A., van Langevelde, P., Kristjansson, M., Maslow, J. N., and Arbeit, R. D. (1995). Persistent and relapsing infections associated with smallcolony variants of Staphylococcus aureus. Clin. Infect. Dis. 20, 95-102. doi: 10.1093/clinids/20.1.95

Proctor, R. A., von Eiff, C., Kahl, B. C., Becker, K., McNamara, P., Herrmann, M., et al. (2006). Small colony variants: a pathogenic form of bacteria that facilitates persistent and recurrent infections. Nat. Rev. Microbiol. 4, 295-305. doi: 10.1038/nrmicro1384

Roggenkamp, A., Sing, A., Hornef, M., Brunner, U., Autenrieth, I. B., and Heesemann, J. (1998). Chronic prosthetic hip infection caused by a smallcolony variant of Escherichia coli. J. Clin. Microbiol. 36, 2530-2534.

Sauders, B. D., Wiedmann, M., Desjardins, M., Fenlon, C., Davenport, N., Hibbs, J. R., et al. (2001). Recurrent Listeria monocytogenes infection: relapse or reinfection with a unique strain confirmed by molecular subtyping. Clin. Infect. Dis. 33, 257-259. doi: 10.1086/321821

Segal, A. W. (2005). How neutrophils kill microbes. Annu. Rev. Immunol. 23, 197-223. doi: 10.1146/annurev.immunol.23.021704.115653

Seifert, H., Von Eiff, C., and Fätkenheuer, G. (1999). Fatal case due to methicillinresistant Staphylococcus aureus small colony variants in an AIDS patient. Emerg. Infect. Dis. 5, 450-453. doi: 10.3201/eid0503.990319

Sendi, P., and Proctor, R. A. (2009). Staphylococcus aureus as an intracellular pathogen: the role of small colony variants. Trends Microbiol. 17, 54-58. doi: 10.1016/j.tim.2008.11.004

Shaughnessy, L. M., and Swanson, J. A. (2007). The role of the activated macrophage in clearing Listeria monocytogenes infection. Front. Biosci. 12:2683-2692. doi: 10.2741/2364

Sousa, A. M., Machado, I., and Pereira, M. O. (2012). "Phenotypic switching: an opportunity to bacteria thrive," in Science against Microbial Pathogens: Communicating Current Research and Technological Advances, ed. A. MéndezVilas (Berlin: Springer), 252-262.

Temple, M. E., and Nahata, M. C. (2000). Treatment of listeriosis. Ann. Pharmacother. 34, 656-661. doi: 10.1345/aph.19315

Tuchscherr, L., Medina, E., Hussain, M., Völker, W., Heitmann, V., Niemann, S., et al. (2011). Staphylococcus aureus phenotype switching: an effective bacterial strategy to escape host immune response and establish a chronic infection. EMBO Mol. Med. 3, 129-141. doi: 10.1002/emmm.201 000115

Vijh, S., and Pamer, E. G. (1997). Immunodominant and subdominant CTL responses to Listeria monocytogenes infection. J. Immunol. 158, 3366-3371.

von Eiff, C., Bettin, D., Proctor, R. A., Rolauffs, B., Lindner, N., Winkelmann, W., et al. (1997). Recovery of small colony variants of Staphylococcus aureus following gentamicin bead placement for osteomyelitis. Clin. Infect. Dis. 25, 1250-1251. doi: 10.1086/516962

Walloschke, B., Fuhrmann, H., and Schumann, J. (2010). Macrophage cell line RAW264.7 but not P-388D1 is an appropriate in vitro-model for studying oxidative burst as well as cytokine production in context of fatty acid enrichment. Cell. Immunol. 262, 58-61. doi: 10.1016/j.cellimm.2009.12.008

Winslow, D., Damme, J., and Dieckman, E. (1983). Delayed bactericidal activity of beta-lactam antibiotics against Listeria monocytogenes: antagonism of 
chloramphenicol and rifampin. Antimicrob. Agents Chemother. 23, 555-558. doi: 10.1128/AAC.23.4.555

Wulff, G., Gram, L., Ahrens, P., and Vogel, B. F. (2006). One group of genetically similar Listeria monocytogenes strains frequently dominates and persists in several fish slaughter- and smokehouses. Appl. Environ. Microbiol. 72, 43134322. doi: 10.1128/AEM.02288-05

Zahra, M., Ferreri, M., Alkasir, R., Yin, J., Han, B., and Su, J. (2013). Isolation and characterization of small-colony variants of ornithobacterium rhinotracheale. J. Clin. Microbiol. 51, 3228-3236. doi: 10.1128/JCM. 01337-13
Conflict of Interest Statement: The authors declare that the research was conducted in the absence of any commercial or financial relationships that could be construed as a potential conflict of interest.

Copyright (1) 2016 Curtis, Gram and Knudsen. This is an open-access article distributed under the terms of the Creative Commons Attribution License (CC BY). The use, distribution or reproduction in other forums is permitted, provided the original author(s) or licensor are credited and that the original publication in this journal is cited, in accordance with accepted academic practice. No use, distribution or reproduction is permitted which does not comply with these terms. 